\title{
An Assessment on Brightness Preservation Techniques over Digital Image Processing
}

\author{
Vaishali Ahirwar \\ Department of Computer \\ Science \& Engineering \\ RITS, Bhopal
}

\author{
Himanshu Yadav \\ Department of Computer \\ Science \& Engineering \\ RITS, Bhopal
}

\author{
Anurag Jain \\ Department of Computer \\ Science \& Engineering \\ RITS, Bhopal
}

\begin{abstract}
Digital image processing forms core research area with in computer science disciplines. Rapid growth of image processing technologies has been used digital images more and more prominent in our daily life. Brightness preservation is a technique of improving the image brightness so that the limitations contained in these images is used for various applications in a better way. The paper presents a review on using hybrid transformation means used combination of two transformation techniques first, curvelet transformation is used to identify the bright regions of the original image and second, discrete wavelet transformation used for reduce Noise and compressed the image for improve the quality of images and then the histogram equalization method is used to enhance the image brightness. Histogram Equalization technique is one of the most popular methods for image enhancement due to its simplicity and efficiency. This is a review on these methodologies by which it is possible to preserve the brightness more efficiently.
\end{abstract}

Keywords: image processing, Brightness Preservation, Hybrid transformation, Histogram Equalization

\section{INTRODUCTION}

The utility of digital images are very much common for all kind of display gadgets. Unfortunately, the input images that are captured by these devices are sometimes not really in good brightness and contrast. Therefore, a process known as digital image enhancement is normally required to increase the quality of these low brightness images. The objective of image enhancement techniques is to improve a quality of an image such that enhanced image is better than the original image. Several image enhancement techniques have been proposed in both spatial and transform domains. In the spatial domain techniques, intensity values of images have been modified whereas in the transform domain techniques, transform domain coefficients are modified, typically, scaled [1].Image enhancement produces an output image that subjectively looks better than the original image by changing the pixel's intensity of the input image [2]. The reason of image enhancement is to improve the interpretability or perception of information contained in the image for individual viewers, or to make available an improved input for other automated image processing systems. It plays an important role in the use of images in various applications like cancer and tumor detection, medical image processing, radar image processing etc. Many such techniques have been proposed such as brightness preserving bi-histogram equalization (BBHE) [4], dualistic sub-image histogram equalization (DSIHE) [5], minimum mean brightness error bihistogram equalization (MMBEBHE) [6], recursive mean separate histogram equalization (RMSHE) [7], Recursively
Separated and Weighted Histogram Equalization (RSWHE) [8], multi-histogram equalization (MMLSEMHE) [9], brightness preserving dynamic histogram equalization (BPDHE) [10], and Image Dependent Brightness Preserving Histogram Equalization (IDBPHE). The proposed method includes identifying the bright regions using Discrete Wavelet Transform (DWT) [1].

\section{IMAGE PROCESSING}

Image processing is a method to convert an image in to digital form using different operation on it, in order to get an enhanced image. Purposes of image processing are following:-

1. Visualization - Observe the objects that are not visible.

2. Image sharpening and restoration - To create a better image.

3. Image retrieval - Seek for the image of interest.

4. Measurement of pattern - Measures various objects in an image.

5. Image Recognition - Distinguish the objects in an image

\section{HYBRID TRANSFORMATION}

This paper processes a hybrid transformation technique ,where the identify of bright regions of image by curvelet transformation and for image compression and reduced noise using discrete wavelets transformation the advantages of this approach include the potential for improving the brightness and efficiency of image.

\subsection{Curvelet Transformation}

The Curvelet transform is a higher dimensional generalization of the Wavelet transform designed to represent images at different scales and different angles. Curve lets enjoy two unique mathematical properties, namely :-

- Curved singularities can be well approximated with very few coefficients and in a non-adaptive manner - hence the name"curvelets".

- Curvelets remain coherent waveforms under the action of the wave equation in a smooth medium.

The Curvelet Transform includes four stages

\subsubsection{Sub-band decomposition}

$$
\mathrm{f} \mapsto\left(\boldsymbol{P}_{0} f, \Delta_{1} f, \Delta_{2} f, \ldots\right)
$$

1. Dividing the image into resolution layers.

2. Each layer contains details of different frequencies: $P_{0}-$ Low-pass filter. 


$$
\Delta_{1}, \Delta_{2} \ldots=\text { Band - pass (high-pass) filters. }
$$

3. The original image can be reconstructed from the sub-bands:

$$
\mathrm{f}=P_{0}\left(P_{0} f\right)+\sum \Delta_{5}\left(\Delta_{s} f\right)
$$

4. Energy preservation

$$
\|f\|_{2}^{2}=\|P Q f\|_{2}^{2}+\sum_{S}\|\Delta S f\|_{2}^{2} .
$$

\subsubsection{Smooth partitioning}

A grid of dyadic squares is defined:

$$
\mathcal{Q}_{(S, K 1, K 2)}=\left[\frac{K 1}{2^{5}}, \frac{K 1-1}{2^{S}}\right] \times\left[\frac{K 2}{2^{5}}, \frac{K 2+1}{2^{S}}\right] \in \mathcal{Q}_{S}
$$

$\mathrm{Q}_{s}$ - All the dyadic squares of the grid.

Let $w$ be a smooth windowing function with 'main' support of size $2^{-s} \times 2^{-s}$. For each square, $w_{Q}$ is a displacement of $w$ localized near $Q$. Multiplying $\Delta_{s} f$ with $w_{Q}\left(\forall Q \in \mathrm{Q}_{s}\right)$ produces a smooth dissection of the function into 'squares'.

$$
h_{Q=W_{Q}} \cdot \Delta_{S} f
$$

\subsubsection{Renormalization}

Renormalization is centering each dyadic square to the unit square $[0,1] \times[0,1]$.For each $Q$, the operator $T_{Q}$ is defined as:

$$
\left(T_{Q} f\right)(X 1, X 2)=2^{5} f\left(2^{S}{ }_{X 1-K 1}, 2^{S}{ }_{X 2-K 2}\right)
$$

Each square is renormalized

$$
G_{Q}=T_{Q}^{-1} \cdot \mathcal{H}_{Q}
$$

\subsubsection{Ridgelet analysis}

Ridgelet are an orthonormal set $\left\{\rho_{\lambda}\right\}$ for $L^{2}\left(\Re^{2}\right)$. Divides the frequency domain to dyadic coronae $|\xi| \in\left[2^{s}, 2^{s+1}\right]$.

In the angular direction, samples the $s$-th corona at least $2^{s}$ times. In the radial direction, samples using local wavelets.

The Ridgelet element has a formula in the frequency domain:

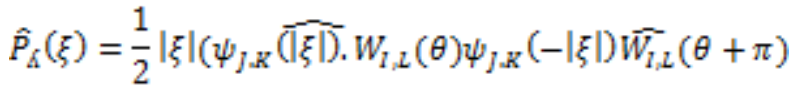

Where, $\omega_{i, l}$ are periodic wavelets for $[-\pi, \pi] . I$ is the angular scale and $l \in\left[0,2^{i-1}-1\right]$ is the angular location. $\psi_{j, k}$ are Meyer wavelets for $\boldsymbol{J}$ is the Ridge let scale and $k$ is the Ridge let location.

First generation curvelet transform is a multiscale directional transform which has been designed to represent edges and other singularities along curves effectively. It enables directional analysis of images in different scales. Second generation curvelet transform called the discrete curvelet transformation (DCT) have been proposed with less complexity and fast computation. It has two different decompositions namely unequispaced DCT (UDCT) and the wrapping DCT (WDCT). In UDCT, the curvelet coefficients are found by irregularly sampling the Fourier coefficients of images. In WDCT, series of translations and wraparound technique are used.

\subsection{Discrete wavelet transform}

Wavelet transform is used to image compression which is involves reducing the typically massive amount of data needed to represent image. WT is also used to detect the edge information, enhancement, fusion, de-noise and so on. WT provide a time frequency representation of the signal.

The wavelet series is just a sampled version of continuous wavelet transformation. DWT which is based on sub band coding is formed to yield fast computation of wavelet transformation. it is easy to implement and reduce to computation time and resources required. The DWT is computed by successive low pass and high pass filtering of the discrete time domain signal.

In DWT signal is denoted by $\mathrm{I}[\mathrm{n}]$ sequence ,where $\mathrm{n}$ is integer, low pass filter is denoted by LP and high pass filter is denoted by HP and $\downarrow \square$ denotes sub sampling.at each level HP produces detail information $\mathrm{Di}(\mathrm{n})$.while the LP associated with scaling function produce approximation $A(n)$. If a lowpass threshold filters out most of high-frequency coefficients, much noise will be deleted, and the image will become smoother. But, the image will also become blurring, because many detail characters are also deleted. It means that it is not enough to distinguish noise ingredient from detail ingredient simply according to the frequency of WT.DWT are defined as follows:-an input image I (n), let I(X, Y) represent be a 2D image and HP and LP be wavelet analysis filters, and then the $\mathrm{N}$ level wavelet transform of image can be described as

$$
\operatorname{In}\left(X_{y} Y\right)=\sum_{m, n \in z} H P(m) L P(n) A n-1\left(2 x-m_{v} 2 y-\right.
$$

n)

$$
\begin{aligned}
& \mathrm{D} 1 \mathrm{n}(\mathrm{x}, \mathrm{y}) \\
& =\sum_{m, n \in z} H P(m) L P(n) A n-1\left(2 x-m_{s} 2 y-n\right) \\
& \mathrm{D} 2 \mathrm{n}(\mathrm{x}, \\
& \mathrm{y})=\sum_{m_{y} n \epsilon_{z}} H P(m) L P(n) A n-1\left(2 x-m_{s} 2 y n\right) \\
& D 3 n\left(x_{x} y\right)=\sum_{m, n \in z} H P(m) L P(n) A n-1\left(2 x-m_{s} 2 y-n\right)
\end{aligned}
$$

The wavelet transform has a reconstruction algorithm which can be described as

$$
\begin{aligned}
& A n-1(x, y)=4 *\left[\sum_{m n \in z} \widehat{H P}(m) L P(n) A \bar{n}\left(\frac{x-m}{2}, \frac{y-n}{2}\right)\right. \\
& \text { ] }
\end{aligned}
$$

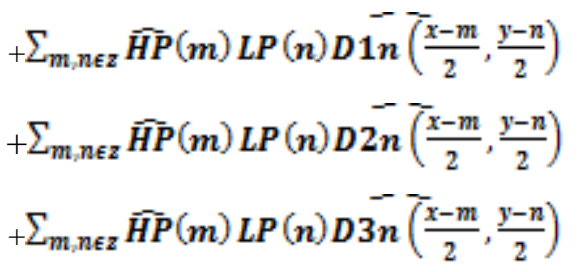

Classification of wavelet is two types, 1.orthogonal and 2.biorthogonal.orthogonal filter coefficients are real numbers. The filters are of the same lengths but not symmetric .LP and HP filters are related to each others

$$
H P(Z)=Z^{-N} L P\left(-Z^{-1}\right)
$$

This property is useful in many signal and image processing applications. They have regular structure which leads to easy implementation and scalable architecture. In the bi-orthogonal wavelet filter, low pass filter is always symmetric while the 
high pass filter is either symmetric or anti-symmetric the coefficients of these filter are either real number or integer. the linear phase bi-orthogonal filters are the most popular filter for data compression applications.

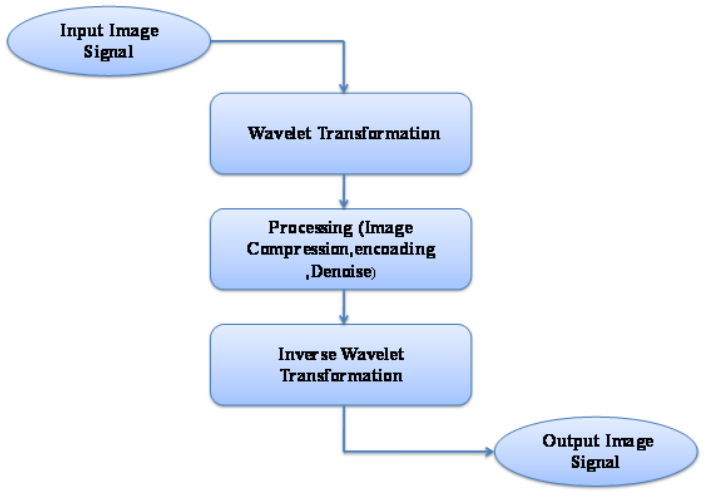

Fig.-2 image processing using wavelet transformation

In fig.-2 show the process of image signal using wavelet transformation. In processing steps involves compression, encoding, de-noising etc.The processed signal is either stored or transmitted. For most compression applications, processing involves quantization and entropy coding to yield a compressed image. During this process, all the wavelet coefficients that are below a chosen threshold are discarded. These discarded coefficients are replaced with zeros during reconstruction at the other end. To reconstruct the signal, the entropy coding is decoded, then quantized and then finally Inverse Wavelet Transformed.

\subsubsection{Discrete Wavelet Architecture}

1-D wavelet scheme which transform along the rows and then a 1-D wavelet transform along the columns (shown in fig.3).Two main methods exist for the implementation of 1DDWT: 1.The traditional convolution-based implementation and 2.the lifting-based implementation. 1-D WT architectures can be classified into many types, the main ones are: space multiplexed, systolic array, time multiplexed, folded, and digit-serial. There are techniques for improving these designs, which include lattice, pipelining/register networking, combined DWT and IDWT, and approximating results.

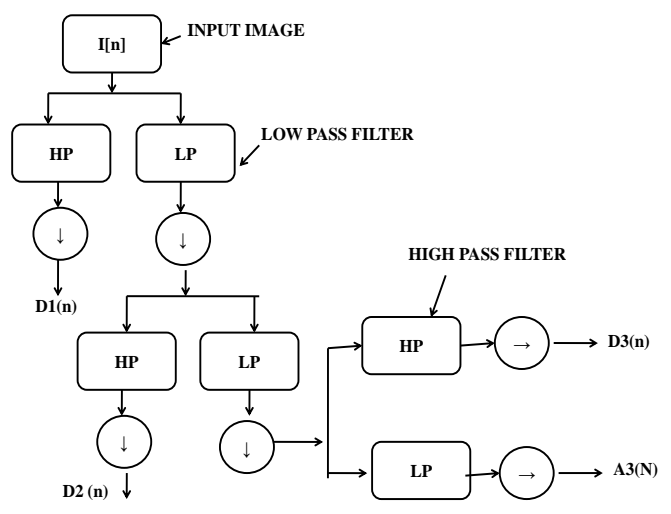

Fig.3:-1D wavelet transformation
The basic idea of 2-D architecture is similar to 1-D architecture. A 2-D DWT can be seen (in fig.4) as a 1-D wavelet scheme which transforms along the rows and then a 1-D wavelet transform along the columns.

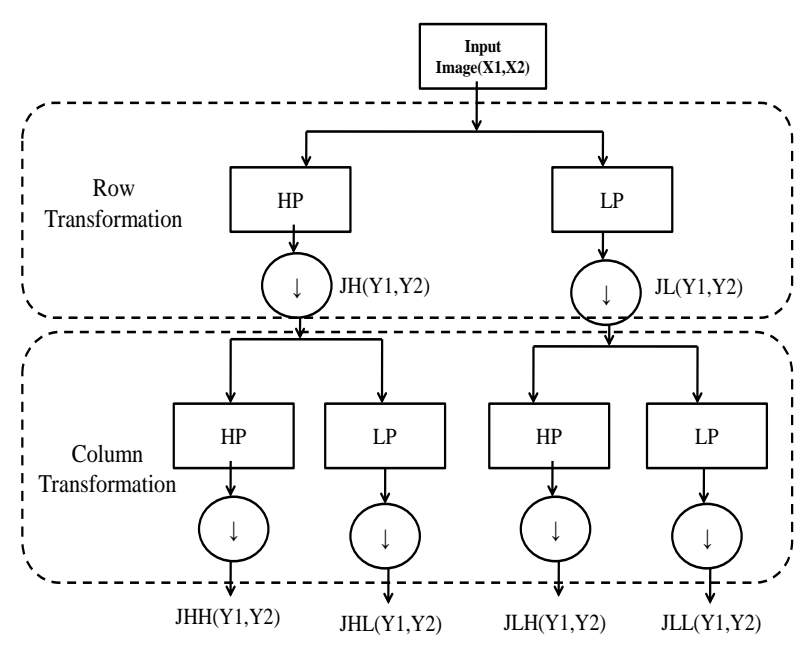

Fig.4:-2D wavelet transformation

The 2-D DWT operates in a straightforward manner by inserting array transposition between two 1-D. The rows of the array are processed first with only one level of decomposition. This essentially divides the array into two vertical halves, with the first half storing the average coefficients, while the second vertical half stores the detail coefficients. This process is repeated again with the columns, resulting in four sub-bands HH, HL, LH, and LL.The 3D DWT (shown in fig.5) can be considered as a combination of three 1D DWT in the $x, y$ and $z$ directions. A preliminary work in the DWT processor design is to build 1D.

DWT modules, which are composed of high-pass and lowpass filters that perform a convolution on filter coefficients and input pixels. After a one-level of 3D discrete wavelet transform, the volume of frame data is decomposed into HHH, HHL, HLH, HLL, LHH, LHL, LLH and LLL signals.

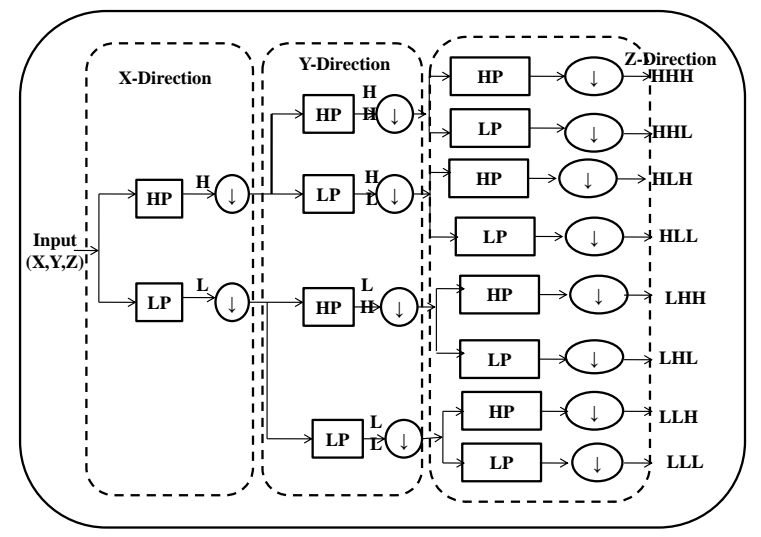

Fig.5:-3D wavelet transformation 


\section{HISTOGRAM EQUALIZATION}

There are many image enhancement techniques that have been proposed and developed, the most popular method being Histogram Equalization. This technique is one of the most popular methods for image enhancement due to its simplicity and efficiency. Histogram is a plot of frequency of occurrences of an event. Histogram of image provide a global description of enormous information is obtained it is a spatial domain technique. Histogram of an image represents relative frequency of occurrence of various gray levels.

\section{- Input Image}

- Histogram Processing of Image

- Apply Histogram Equalization

- Histogram Matching

- Enhance Input Image

Fig.6:-process of histogram equalization

\section{Histogram processing of image}

Histogram can be plotted in two ways:-

1. $\mathrm{X}$-axis has gray level and $\mathrm{Y}$-axis has number of pixels in each gray level.

2. X-axis has gray level and Y-axis has probability of occurrence of gray level.

$$
P(\mu k)=\frac{\eta k}{\eta}
$$

Where $\boldsymbol{\mu k}$-gray level, nk-number of pixel in kth gray level.ntotal number of pixel in an image. Advantages of second method maximum value plotted will always be 1 . white $=1$, black $=0$.

\section{Histogram equalization}

Linear stretching is a good technique but not perfect since the shape remain the same. Most of the times need a flat histogram. It can't be achieved by histogram stretching thus new technique of histogram equalization came in to use, perfect image is one where all gray level have equal no of pixel. Search for a transform that converts any random histogram in to flat histogram

$$
\mathbf{S}=\mathbf{T}(\mathbf{r})
$$

To find ' $\mathrm{T}$ ' which produces equal values in each gray level, the transform should satisfy following conditions:-

1. $T(\mathrm{r})$ must be single value and monotonically increasing in the interval $\mathbf{0} \leq \mathbf{r} \leq \mathbf{1}$.

\section{2. $0 \leq T(r) \leq 1$ for $0 \leq r \leq 1$ and $0 \leq S \leq 1$ for $0 \leq r \leq 1$}

Here, range of $r$ is $[0,1]$.

\section{Histogram matching}

HE automatically determines Transformation function seeking to produce an output image with a uniform histogram. Another method is to generate an image is having a specified histogram - histogram matching:

Find the histogram $T(r)$ of the input image and determine its equalization transformation

$$
S=T(r)=(L-1) \int_{0}^{r} p(r) W d w
$$

Use the specified pdf $P_{Z}(r)$ of the output image to obtain the Transformation function:

$$
G(z)=(L-1) \int_{0}^{z} p_{z}(t) d t=S
$$

Find the inverse transformation $z=G-1(s)-$ the mapping From $s$ to $z$ :

$$
Z=G^{-1}[T(r)]=G^{-1}(S)
$$

Obtain the output image by equalizing the input image first then for each pixel in the equalized image, perform the inverse mapping to obtain the corresponding pixel of the output image.

\section{Types of histogram}

Four basic image types and their histogram (in fig.1, 2, 3, 4)

1. Dark image 2 light image 3. Low contrast image 4. High contrast image
1.
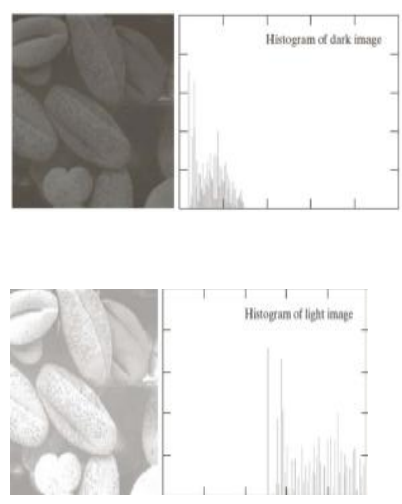

2.
3.
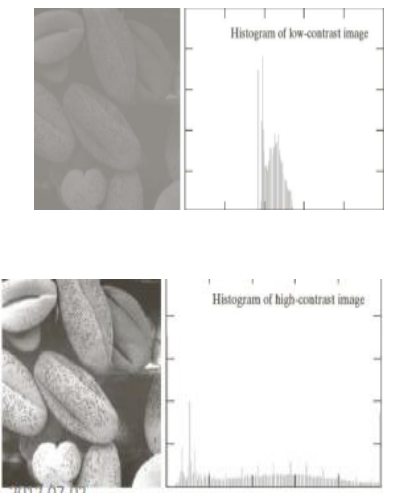

4.
Histogram equalization (HE) [2] is a simple and effective contrast enhancement technique which distributes pixel values uniformly such that enhanced image have linear cumulative histogram. The HE technique is a global operation hence; it does not preserve the image brightness [3].

According to Kim [4], one drawback of the histogram equalization can be found on the fact that the brightness of an image can be altered after the histogram equalization, just because of to the flattening property of the histogram equalization. Therefore, it is hardly utilized in consumable electronic products such as TV where preserving original 
input brightness may necessary in order not to introduce unnecessary visual deterioration [4]. Kim proposed a technique whereby the input histogram is divided into two sub-histograms on the basis of its mean value. The main motive behind this technique was to preserve the brightness of the image while enhancing its contrast.

Wan et. al. [5] proposed Dualistic sub-image histogram equalization (DSIHE). They also do the same thing that BBHE does but the criterion for separation of histograms is median value. They also proposed another technique namely Recursive Mean Separate Histogram Equalization (RMSHE) [7], where the histogram is recursively partitioned based on local mean values and the number of sub-histograms $\left(2^{r}\right)$ is given by user. Minimum Mean Brightness Error Bi-Histogram Equalization (MMBEBHE) technique was proposed by Chen and Ramli [6]. In this technique the histogram is partitioned based on a threshold level which is equivalent to minimized difference between the input mean and output mean. In case of preserving the brightness of original image this method is better than BBHE and DSIHE Wang et. al. [10] proposed a technique called Brightness Preserving Histogram Equalization with Maximum Entropy (BPHEME). The idea behind their technique was to find the target histogram that maximizes the entropy, keeping in view that brightness of original image is preserved, and then apply histogram transformations to transform the original histogram to the targeted one. The results showed that this technique is better than BBHE, DSIHE and MMBEBHE Multi-histogram equalization (MMLSEMHE) methods were proposed by Menotti et. al. [8]. According to them, though bi-HE methods preserve the brightness of the original image but, the output image may not look as natural as the original image. They proposed a technique which on one hand preserves the brightness of the input image and on the other hand generated images with natural appearances. Later in the same year, Ibrahim et. al. proposed their method for preserving brightness entitled preserving dynamic histogram equalization (BPDHE) [9], in which the histogram is first subjected to 1-D Gaussian filter and then it is sub-partitioned into a number of sub-histograms based on its local maximums. Each subhistogram is then equalized separately. Hussein et. al. [11] proposed a technique called Minimum Mean Brightness Error Dynamic Histogram Equalization (MMBEDHE) whereby, the input image is divided into a number of sub-images and then the classical HE is applied to each of them. The absolute average error using this technique was calculated to be very less as compared to since then existing techniques [11].

In the same year, Xia et. al. [12] came up with their technique for image enhancement known as "An Adaptive Image Enhancement Technique Based on Image Characteristic". In this technique, the actual or original image is primary subjected to Laplace Filter which is a spatial high-pass filter. Based on its output, the first-order classifying of the image is done. Here the image is smoothened using low-pass filter and the edges are sharpened using high-pass filter. At the end, HE is applied to it [13].

Continuing with the research Demirel et. al. [13] proposed a new method for enhancement of satellite images contrast called Satellite Image Contrast Enhancement Using Discrete Wavelet Transform and Singular Value Decomposition. Their method was based on Discrete Wavelet Transform (DWT) and singular-value decomposition. They first applied DWT to input image to divide it into four frequency sub-bands, then used singular value decomposition and then again applied inverse DWT to reconstruct the image. Their technique showed better results than conventional BPDHE [10].

In the same year, P. Rajavel came up with his proposed algorithm for brightness preservation called Image Dependent Brightness Preservation Histogram Equalization (IDBPHE) [1] .In this technique the bright regions of images are identified using Curvelet Transform and then histogram of original image is modified with respect to the histogram of the identified regions. Continuations with literature review, a new technique was proposed in [15] for image brightness preservation that used transformation functions that utilize both the global as well as local information contents of the image. The name to this proposed technique was given as Brightness Preserving Image Contrast Enhancement Using Weighted Mixture of Global and Local Transformation Functions [14].Sheet et. al. proposed a new technique in November 2010 called Brightness Preserving Dynamic Fuzzy Histogram Equalization (BPDFHE) [15] which was the modified version of BPDHE [10] whereby the fuzzy statistics of digital images were used to represent and process them. The results showed that this method was better than BPDHE for preserving the brightness and enhancing the contrast of the image.

\section{CONCLUSION}

In this paper a review of Image Dependent Brightness Preserving Histogram Equalization has been discussed for the image brightness enhancement using Discrete Wavelet Transform. The analysis shows that the image brightness through discrete wavelet transformation (DWT) provides better results as compared to the other wavelet transformations like Curvelet Transform (as shown by various researchers). To continuation with this we also observed that Brightness Preservation using Hybrid Transformation of Images using Histogram Equalization are enhanced the result drastically. It is also observed that various researchers are using single form of transforms. It may be possible to enhance these results using hybrid transformation pattern in Histogram Equalization for low brighten images. According to literature review we proposed a new scheme for preserving brightness of images using hybrid transformation scheme with Histogram Equation.

\section{REFERENCES}

[1] P. Rajavel, "Image Dependent Brightness Preserving Histogram Equalization", IEEE Transactions on Consumer Electronics, Vol. 56, No. 2, May 2010.

[2] Rafael C. Gonzalez, and Richard E. Woods, "Digital Image Processing", 2nd edition, Prentice Hall, 2002.

[3] N. Sengee and H. K. Choi "Brightness Preserving Weight Clustering Histogram Equalization", IEEE Transactions on Consumer Electronics, Vol. 54, No. 3, AUGUST 2008.

[4] Yeong-Taeg Kim, "Contrast enhancement using brightness preserving bi-histogram equalization," IEEE Transactions on Consumer Electronics, vol. 43, no. 1, pp. 1-8, Feb. 1997.

[5] Yu Wan, Qian Chen and Bao-Min Zhang, "Image enhancement based on equal area dualistic sub-image histogram equalization method," IEEE Transactions on Consumer Electronics, vol. 45, no. 1, pp. 68-75, Feb. 1999. 
[6] Soong-Der Chen, and Abd. Rahman Ramli, "Contrast enhancement using recursive mean-separate histogram equalization for scalable brightness preservation," IEEE Transactions on Consumer Electronics, vol. 49, no.4, pp. 1301-1309, Nov. 2003.

[7] Mary Kim and Min Gyo Chung, "Recursively Separated and Weighted Histogram Equalization for Brightness Preservation and Contrast Enhancement", IEEE Transactions on Consumer Electronics, vol. 54, no. 3, pp. 1389-1397, August 2008.

[8] D. Menotti, L. Najman, J. Facon, and A. A. Araujo, "Multi-Histogram Equalization Methods for Contrast Enhancement and Brightness Preserving," Recursively Separated and Weighted Histogram Equalization For Brightness Preservation and Contrast Enhancement, vol. 53, no. 3, pp. 1186- 1194, Aug 2007.

[9] H. Ibrahim and N. S. P. Kong, "Brightness Preserving Dynamic Histogram Equalization for Image Contrast Enhancement," Recursively Separated and Weighted Histogram Equalization For Brightness Preservation and Contrast Enhancement, vol. 53, no. 4, pp. 1752-1758, Nov. 2007.

[10] Chao Wang and Zhongfu Ye, "Brightness Preserving Histogram Equalization with Maximum Entropy: A Variational Perspective", IEEE Transactions on Consumer Electronics, vol. 51, no. 4, pp. 1326-1334, November 2005.
[11] Md. Foisal Hussein and Mohammad Reza Alsharif, "Minimum Mean Brightness Error Dynamic Histogram Equalization For Brightness Preserving Image Contrast Enhancement", International Journal of Innovative Computing, Information and Control, vol. 5, no. 10 (A), pp. 3249-3260, October 2009.

[12] Xia odong Xie, Zaifeng Shi, Wei Guo, Suying Yao, “An Adaptive Image Enhancement Technique Based on Image Characteristic", $2^{\text {nd }}$ International Congress on Image and Signal Processing, CISP'09, pp. 1-5, Oct. 2009.

[13] Hasan Demirel, Cagri Ozcinar, and Gholamreza Anbarjafari,"Satellite Image Contrast Enhancement Using Discrete Wavelet Transform and Singular Value Decomposition", IEEE Geosciences' and Remote Sensing Letters, vol. 7, no. 2, pp. 333-337, April 2010.

[14] Hasanul Kabir, Abdullah Al-Wadud, and Oksam Chae, "Brightness Preserving Image Contrast Enhancement Using Weighted Mixture of Global and Local Transformation Functions", The International Arab Journal of Information Technology, vol. 7, no. 4, October 2010.

[15] Debdoot Sheet, Hrushikesh Grad, Amit Suveer, Manjunatha Mahadevappa, and Jyotirmoy Chatterjee, "Brightness Preserving Dynamic Fuzzy Histogram Equalization", IEEE Transactions on Consumer Electronics, vol. 56, no. 4, pp. 2475-2480, November 2010. 